\title{
Application and Research of GIS in Optimizing Power Grid Dispatchment XIA TIAN
}

\author{
School of Electrical and Electronic Engineering Institute, North China Electric Power University, \\ Baoding 071000, China \\ 1319549621@qq.com
}

Keywords: GIS, Optimizing power grid dispatchment, Application

\begin{abstract}
The power load gradually increases with the development of the city construction which highlights the optimization of power grid dispatchent, and in which a new computer system, the GIS short for Geographic information system plays an important role. This text on basis of introducing GIS conotation, elaborates its application and function in dispatching optimization of power grid..
\end{abstract}

\section{Introduction:}

Power grid dispatching is to guarantee regional power demand balance, in which the GIS as product of modern science and technology development is introduced. As a computer system of geographical information acquisition, storage, analysis, display and application, GIS plays an important role in reasonable dispatchment for enterprise and household power demand.

\section{Connotation of Geographical Information}

Theoretically speaking, GIS mainly refers to technical system, which acquisition, storage, management, operation, analysis display and description of the geographic distributing data of all earth surface spaces in the atmosphere under support of modern computer devices and related software system, to further solve complex planning and management[1]. In essence, GIS goes across the elementary science of geoscience, information science and spatial science, whose role is important in transportation, mining survey and power grid dispatch.

\section{The application of GIS in optimizing power grid dispatchment}

Currently, the constantly city planning lead to emergence of issues such as multiple grid nodes, decentralized devices etc. In this circumstance, the powder distributing GIS, which allows for a intuitive management of the power grid dispatchment is introduced after repeatedly selections in order to further improve the power grid's operating level and efficiency. The writer elaborates GIS application in the dispatching optimization of power grid in the text below.

\subsection{Query function application of Geographic information}

query function of geographic information is the elementary application in dispatching optimization of power grid, which in essence is the query for geographical circumstance. We manipulate regional query via computer information system. Geographic information query function, to a large degree is to realize basic map operations, what are daily mentioned amplification, diminish, translation, layer control, eagle eye, ranging and full picture display of the map[2]. Key words query is mainly utilized in power grid GIS, in which process, technicians will set different query conditions based on their regions. Here we set an example, the Tanshan Fengtai District's Power Authority could input key words "Xugezhuang street" "Fengnan Town" to inquire the power consumption respectively for both these areas; however, if input specific places or enterprises such as "Beiliuhe", then the total power consumption will be get, then the PA shall know the load situation.

It should be noticed that to the power GIS query, its inquiry methods includes enquiry mode, circular query and rectangle query etc, which achieves good effects to different applications of the 
PA and also plays an important role in dispatching optimization of power grid.

\subsection{Application of dispatching tasks and historical records}

It is important to do a good job in dispatching tasks and historical records for dispatching optimization of power grid. A database exists respectively in the GIS software programming and designing, which database mainly refers to combination of SDE of Arc GIS and Oracel database to grid spatial database[3]. While the dispatching tasks and historical records will be entered into this SDE. With the development of information technology, the regularity and accuracy could be gradually summarized, then we could find the available regularity according to necessary historical records to well prepare the future electric power optimization and avoid power shortage.

The dispatching tasks mainly refer to the regional power dispatch, while historical records include working record, operating record, users login records, device records etc. Doing a good job in power grid dispatching tasks and historical records offer great advantages on past electricity quantity consume query for current and future working staffs.

\subsection{Fault handling application}

As an important application in dispatching optimization of power grid, faculty handling, theoretically speaking, is a way of providing solution for power fault in short time. According to writer's experience, the fault handling here mainly refers to the shortest distance from servicing station to fault locations or the best route from location of the overhauling car to fault locations. In this way, the Power authority is able to solve the electric fault with fastest speed to reduce relate loss.

The GIS on the basis of via Dijkstra and Folyd arithmetic dealing with fault handling leads to the result that is its main function is to do spatial analysis. Effective combination of different arithmetic greatly improve spatial analysis ability and its application in electric power is expanded, for example, the GIS could effectively ease "power shortage" via fuzzy neural networks' analysis and summary combined by fuzzy logic and fuzzy neural network.

\subsection{Outage systematic management}

The Outage system management is another application of power grid dispatching optimization. From the viewpoint of GIS, itself in essence is a geographic database. The outage region has to be defined above all in order to do a good job in outage systematic management. Therefore GIS query should be used to make the PA regions relating figure data entity and description properties.

The outage systematic management mainly refers to the PA, after receiving outage complaints from enterprises or personnel, inquiring corresponding locations and scopes in best time and chooses reasonable operating orders and routes via CIS and SCADA function of the GIS. The progress of the handling of these incidents could be obviously displayed and automatically forward to complaints telephone answering system[4]. In addition, the auxiliary power development planning and designing function of GIS could timely avoid the outage, and even if there is places power off, related coordination can be done in best time.

\section{GIS application in optimization for power grid dispatchment}

The electricity power information system plays an important role in optimizing power grid dispatch. The write gives a brief analysis on the GIS function in optimizing power grid dispatchment in the text below.

\subsection{Accuracy improvement of the power grid dispatching works}

GIS can further improve the accuracy of the electricity dispatching works. In practical working operations, the dispatching SCADA data used on daily life ca be stored in GIS database. Therefore we could clearly see dispatching SCADA systematic pictures on the GIS platform, which is I important for working staff to know and grasp the telemetering and telesignal of every $10 \mathrm{KV}$ wires[5]. If the electricity load surpasses certain amount, scarlet letter alarm will appear to drive the accuracy of electricity dispatch.

\subsection{Promoting city power grid planning and development}

GIS can further promote the city grid planning and development. One of the important work for $\mathrm{PA}$ is to complete the city grid planning according to continuously development of the city. 
Remarks on the GIS are usually the base for Working staff, in the process of city grid planning project related grid layout. This layout, firstly its fabrication is improved for there is no need of practical analysis; on the other side, its quality is guaranteed for GIS embody real conditions accurately, therefore the city grid planning\& development will be greatly improved.

GIS plays an important role in power grid dispatching optimization, which in the text above, mainly elaborated from geographic information query application, dispatching tasks and historical records, fault handling application, outage systematic management. In future works, we will according to practical situation update the GIS system to future optimize the power grid dispatchment.

\section{Conclusion}

GIS has been widely used in our country Optimizing Power Grid Dispatchment system. Not only can GIS significantly reduce the workload of power distribution network scheduling, but also improve the efficiency of the distribution network of dispatchers and the accuracy of incident handling and reduce losses due to accidents. Although there are some objective problems, but it has been able to provide a strong distribution network dispatching data and graphics platform , which is the basis for Optimizing Power Grid Dispatchment, and has been working closely with Optimizing Power Grid Dispatchment.One day GIS application will be more to more extensive and in-depth, the prospects are more widely

\section{References}

[1] Wang Weitao, GIS application in the power distribution network [J]; Science and Technology Innovation Herald, 2010(04)

[2] Yu Chao, Zhao Qiang,Study on the Application of WebGIS-power distribution network, Based on ArcGISServer[J]; Computer and Information Technology 2008(06)

[3] Wang Xiaobing, Sun Jiuyun, Overview of the GIS[J]; Geospatial Information,2012(01)

[4] Yang Kun, Xu Quanli, Peng Shuangyun, Cao Yanbo; Study and Development of the GIS for police use in the city based on ArcGISSeveer

[5] Li Bing; GIS application in Electric Power Industry [J]; Brand(Theory Monthly); 2010(Z2) 